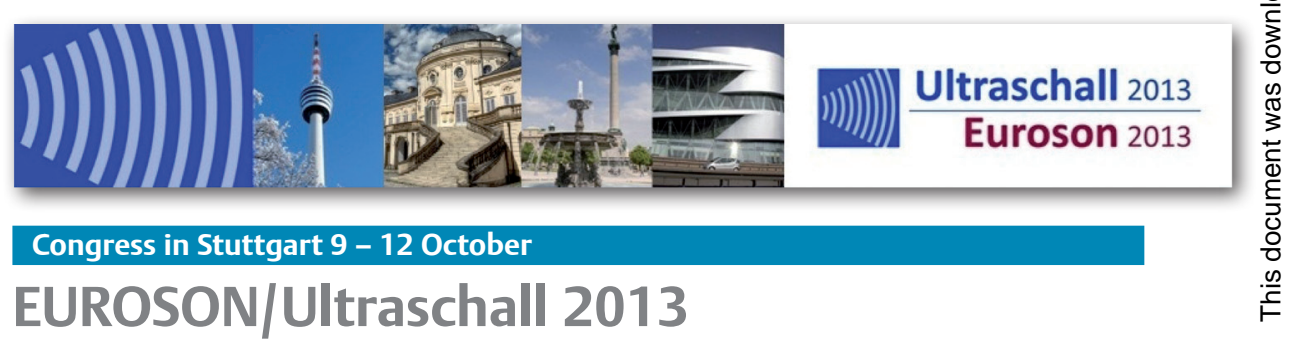

The preparations for the EUROSON/Ultraschall 2013 Congress in Stuttgart 9 - 12 October are moving straightforwardly. The organizers are looking forward to welcoming European specialists and ultrasound scientists as well as clinicians and practitioners in ultrasound. Under the congress motto „Science and Education“ both scientific work as well as the broad spectrum of education and teaching are pointed out to connect the interdisciplinary faculty of European ultrasound providers in the joint meeting of EFSUMB, DEGUM, OEGUM and SGUM. It is definitely this year's main event in ultrasound and the congress of all European experts - it is „our congress“!

\section{Late Breaking News}

The scientific committee was happy to receive more than 350 scientific abstracts mostly of high quality in all offered categories. One of the main topics of the scientific sessions will be ultrasound elastography as a new diagnostic tool. This is now brought to a wide spectrum of scientific evaluation in several fields like liver, other abdominal organs, thyroid, muscu- 
loskeletal, gynecology, breast, small parts, prostate and others. Another top act in actual scientific work is still contrast enhanced ultrasound. Quantification of contrast agents enhancement i.e. in oncology or inflammatory bowel diseases for evaluation of (early) therapeutic response will lead to more influence of sonography in those diagnostic and therapeutic fields.

Ultrasound in Gynecology and Obstetrics as well as in prenatal medicine and breast imaging are big points in this year's joint meeting in Stuttgart. New methods like elastography in these indications are proven and well known tools, like 3D US, are evaluated and discussed. Pediatric sonography with its unique position in imaging for these patients is still of high scientific interest. So there will be more than one session dealing with this topic.

Results of research with ultrasound in neurology, vascular diseases, head and neck as well as in emergency, interventions, endoscopy and in daily clinical practice are presented to the public and discussed in several sessions. Some new techniques will be presented and offer the view to future developments.

Special case reports sessions will give the opportunity to share interesting and educative cases with all participants. This points out the importance of recognizing rare diagnosis as well as the possibility of pitfalls and mystery. Those sessions will be moderated by very experienced specialists and are usually of high interest for all clinicians.

Poster sessions complete the scientific part of the congress offering the possibility of discussion with the scientists and moderators. They are a must for such a congress.

\section{Education program}

Traditionally the Congress starts with the Categorical Courses. These substantial educational courses are focusing on a major ultrasound topic over a series of sessions lasting six hours. They are of interest for the more specialised experts of ultrasound, but from basics to advanced topics.

Throughout the other two and a half days there are almost 80 Refresher Courses of 90 minutes each beside the scientific sessions. They aim to refresh knowledge, reviewing and updating the more basic subjects for those who have not kept up to speed with certain developments or just who need a reminder. There is something of interest for the beginner up to the experts in this field.

\section{Hands on training}

The Ultrasound Learning Center will be open on two days and invites all participants for hands on training in several named sessions and topics. This offers the opportunity to have a close look to Europe's ultrasound experts in real time.
For the first time a special course for medical students connects our future colleagues with our societies.

\section{Program information}

All details and information about the congress are available on the website www. euroson2013.org

See you in Stuttgart.

\section{Andreas Schuler and Fabio Piscaglia}
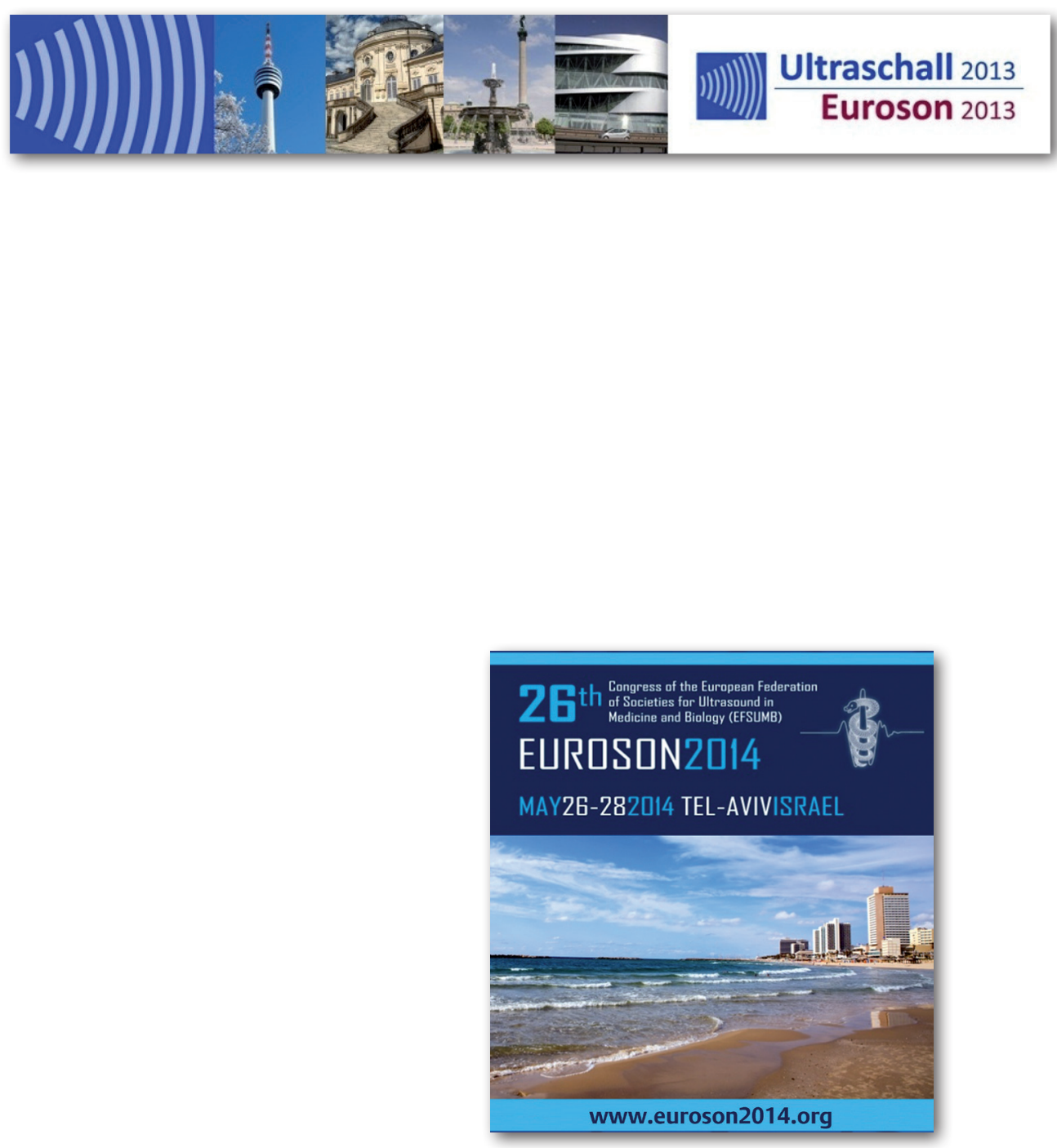